\title{
Dynamics of a beam on elastic foundation - impact of the bilinear characteristic of the foundation upon the propagation of the bending wave
}

\author{
Traian Mazilu ${ }^{1, *}$ and Cristian Ioan Cruceanu ${ }^{2}$ \\ ${ }^{1}$ University Politehnica of Bucharest, Department of Railway Vehicles, Splaiul Independenţei 313, \\ Bucharest, Romania \\ ${ }^{2}$ S.C. Atelierele CFR Griviţa S.A., 359 Calea Griviţei, Bucharest, Romania
}

\begin{abstract}
Rail is an important source of noise in the railway traffic and its acoustic power is depending on the rate of decay of vibration. The main characteristic of the track in terms of the vertical elasticity is the so-called track modulus which results from the load-rail deflection curve. In general, this curve is nonlinear and it can be approximated using piecewise linear function with bilinear characteristic. In this paper, the response of a beam on elastic foundation with bilinear characteristic due to constant and harmonic loads is investigated, pointing out the impact of the foundation characteristic on the rail response and the propagation of the bending waves along the track.
\end{abstract}

\section{Introduction}

The elastic characteristics of the wheels can influence the ride comfort of railway vehicles [1], the wheel-track dynamic loads $[2,3]$ and the rolling noise emissions, with adverse effects on passengers and residents living or working in buildings located in the vicinity of the track [4].

The track modulus is the most important elastic characteristic of the track; the experiments shown that it has a non-linear variation with the wheel load $[5,6]$.

The influence of the bilinear characteristic of the track on the decay rate of the vertical vibrations along the track is studied in this paper, using a continuous model with two elastic layers. Similar approach can be found in ref. [7] where only one elastic layer accounted for.

\section{Mechanical model}

The model presented in Figure 1 is used to study the nonlinear characteristic of the ballast on the propagation of bending waves along the track. For symmetry reasons, the model of the track consists of a rail laid on following elements: rail pads, half-sleepers and ballast bed. The rail is modelled as an infinite and uniform Euler Bernoulli beam, having the

*Corresponding author: trmazilu@yahoo.com 
bending stiffness $E I$, where $E$ is the longitudinal elasticity modulus and $I$ is the inertia momentum of beam's cross section and the mass per unit of length, $m$. The rail's deflection in the $x$ section in relation to the fixed reference system $O x z$ at the $t$ moment is $w(x, t)$.

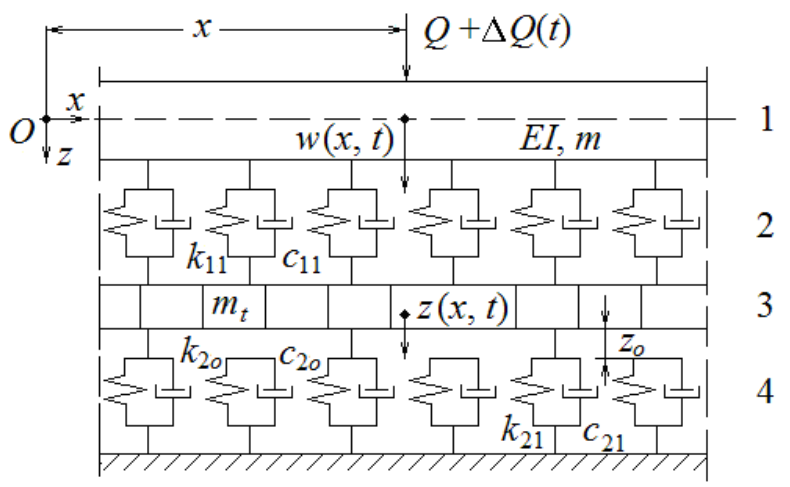

Fig. 1. Mechanical model of the track: 1. Rail; 2. Rail pad; 3. Sleeper; 4. Ballast.

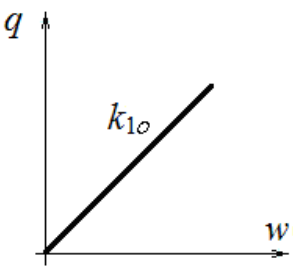

(a)

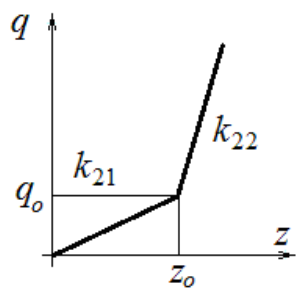

(b)

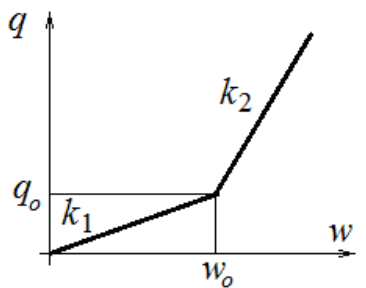

(c)

Fig. 2. Elastic characteristics: (a) Rail pad; (b) Ballast; (c) Track.

The rail pads are modelled using a Kelvin-Voigt type continuous viscoelastic layer having the elastic constant $k_{1 o}$ and the damping constant $c_{1 o}$. The elastic characteristic of the rail pads is presented in the Figure 2 as the distributed force $q$, function of rail displacement $w$. The elastic constant $k_{1 o}$ is results from rail pad's stiffness and the distance between the sleepers. In the same manner the damping constant $c_{1 o}$ is calculated.

The sleepers are modelled as a continuous inertial layer having a mass per unit of length $m_{t}$. The sleepers deflection in the $x$ section of the reference system is $z(x, t)$. The effect of the distance between the sleepers is neglected, so the domain of the model is limited to 6$700 \mathrm{~Hz}$.

The ballast bed is modelled using a Kelvin-Voigt type continuous viscoelastic layer with a bilinear characteristic (Figure 2. b). The ballast's elastic and damping constants are $k_{21}$ and $c_{21}$ when the sleeper's deflection is lower than $z_{o}$, and $k_{22}$ and $c_{22}$ when the sleeper's deflection is higher than $z_{o}$.

The elastic characteristic of the track, respectively the distributed track force $q$ as function of the rail's deflection $w$ is presented in the Figure 2 c. The characteristic is bilinear due to ballast's characteristic. This has two zones: the elastic zone with the elastic constant $k_{1}$ for $w<w_{o}$, and the stiff zone with the elastic constant $k_{2}$ for $w>w_{o}$. The transition point's coordinates between the elastic and stiff zones are $\left(w_{o}, q_{o}\right)$.

Knowing the elastic constants of the track's characteristic, including the coordinates of the transition point between the elastic and stiff zone, as well as the rail pad's elastic constant, the elastic constants of the ballast and the coordinates of the transition point is obtained 


$$
\begin{gathered}
\frac{1}{k_{21}}=\frac{1}{k_{1}}-\frac{1}{k_{1 o}}, \frac{1}{k_{22}}=\frac{1}{k_{2}}-\frac{1}{k_{1 o}} \\
z_{o}=w_{o}-\frac{q_{o}}{k_{1 o}}
\end{gathered}
$$

The force acting on the rail has two components: a static one $Q$ and a dynamic one $Q(t)$, which will be considered harmonic, such that $|\Delta Q(t)|<<Q$.

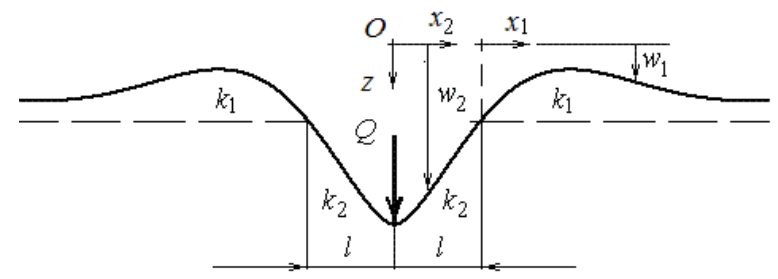

Fig. 3. Explanatory for the boundary conditions under the static load $Q$.

Under the action of the static component, the rail's deformation is not time dependant, as seen from the following equations

$$
\begin{gathered}
E I \frac{\mathrm{d}^{4} w}{\mathrm{~d} x^{4}}+k_{1 o}(w-z)=Q \delta(x), \\
\left(k_{1 o}+k_{21}\right) z-k_{1 o} w=0 \text { if } z<z_{o} \text { or }|x|>l \\
\left(k_{1 o}+k_{22}\right) z-k_{1 o} w=0 \text { if } z \geq z_{o} \text { or }|x| \leq l
\end{gathered}
$$

Where $\delta($.$) is Dirac's function and 2 l$ is the distance between the transition points between the elastic and stiff zones along the beam.

Equations (1-3) can be replaced with

$$
E I \frac{\mathrm{d}^{4} w_{1,2}}{\mathrm{~d} x_{1,2}^{4}}+k_{1,2}=Q \delta(x)
$$

Where $\left|x_{2}\right| \leq l,\left|x_{1}\right|>0, w_{2}\left(x_{2}\right) \geq w_{o}$ and $w_{1}\left(x_{1}\right)<w_{o}$ (Figure 3 ).

Between $w(x)$ and $w_{1,2}\left(x_{1,2}\right)$ there is a following relation

$$
w(x)=\left\{\begin{array}{cc}
w_{2}\left(x_{2}\right)+w_{o}\left(1-k_{1} / k_{2}\right) & \left|x_{2}\right| \leq l, x=x_{2} \\
w_{1}\left(x_{1}\right) & \left|x_{1}\right|>0, x=x_{1}+l \operatorname{sgn}\left(x_{1}\right)
\end{array}\right.
$$

By using the symmetry of the rail's deformation, the boundary conditions are as follows

$$
\begin{gathered}
\frac{\mathrm{d} w_{2}(0)}{\mathrm{d} x_{2}}=0, \frac{\mathrm{d}^{3} w_{2}(0)}{\mathrm{d} x_{2}^{3}}=\frac{Q}{2 E I}, w_{2}(l)=\frac{k_{1}}{k_{2}} w_{o}, w_{1}(0)=w_{o}, \frac{\mathrm{d} w_{2}(l)}{\mathrm{d} x_{2}}=\frac{\mathrm{d} w_{1}(0)}{\mathrm{d} x_{1}}, \\
\frac{\mathrm{d}^{2} w_{2}(l)}{\mathrm{d} x_{2}^{2}}=\frac{\mathrm{d}^{2} w_{1}(l)}{\mathrm{d} x_{1}^{2}}, \frac{\mathrm{d}^{3} w_{2}(l)}{\mathrm{d} x_{2}^{3}}=\frac{\mathrm{d}^{3} w_{1}(l)}{\mathrm{d} x_{1}^{3}}, \mid \lim _{\mid x_{1} \rightarrow \infty} w_{1}\left(x_{1}\right)=0 .
\end{gathered}
$$

The following rail deflection shapes are considered 


$$
\begin{gathered}
w_{2}\left(x_{2}\right)=A_{1} e^{-\alpha_{2} x_{2}} \sin \alpha_{2} x_{2}+A_{2} e^{-\alpha_{2} x_{2}} \cos \alpha_{2} x_{2}+A_{3} e^{\alpha_{2} x_{2}} \sin \alpha_{2} x_{2}+A_{4} e^{\alpha_{2} x_{2}} \cos \alpha_{2} x_{2}, \\
w_{1}\left(x_{1}\right)=A_{5} e^{-\alpha_{1} x_{1}} \sin \alpha_{1} x_{1}+A_{6} e^{-\alpha_{1} x_{1}} \cos \alpha_{1} x_{1} .
\end{gathered}
$$

Where

$$
\alpha_{1,2}=\sqrt[4]{\frac{k_{1,2}}{4 E I}}
$$

From equations (8) - (9), a non-linear 7 equations system is obtained, having the unknowns $A_{1}-A_{6}$ and $l$. The solution can be obtained by using the Newton-Raphson's method. When a variable force $\Delta Q(t)$ is acting on the rail too, the dynamic rail deflection $\Delta w$ and dynamic sleeper displacement, $\Delta z$, are relative to the static equilibrium position due to static load $Q$. Neglecting the effect of the track's vibration around the transition points of the elastic characteristic, the following equations of motion are obtained

$$
\begin{aligned}
& E I \frac{\partial^{4} \Delta w_{1,2}}{\partial x_{1,2}^{4}}+m \frac{\partial^{2} \Delta w_{1,2}}{\partial t^{2}}+c_{1,2} \frac{\partial \Delta w_{1,2}}{\partial t}+k_{1,2}\left(\Delta w_{1,2}-\Delta z_{1,2}\right)=\Delta Q(t) \delta(x), \\
& m_{t} \frac{\partial^{2} \Delta z_{1,2}}{\partial t^{2}}+\left(c_{11}+c_{21,22}\right) \frac{\partial^{2} \Delta z_{1,2}}{\partial t^{2}}+\left(k_{11}+k_{21,22}\right) \Delta z_{1,2}-k_{11} \Delta w_{1,2}=0 .
\end{aligned}
$$

The steady-state harmonic behaviour's solution can be obtained by using the Green's function method [8]. Then, the rail's receptance in the $x$ section due to the harmonic force applied in the $x_{o}$ position, $r\left(x, x_{o}, \omega\right)$, can be computed, as function of pulsation $\omega$.

Finally, the rail decay rate of the vibrations along the rail is calculated

$$
R(x, \omega)=\frac{20}{x} \lg \frac{|r(0,0, \omega)|}{|r(x, 0, \omega)|}[\mathrm{dB} / \mathrm{m}] .
$$

\section{Numerical application}

The results obtained with the above-mentioned model and calculation method are presented in this section. The parameters for the track model are: $m=65 \mathrm{~kg} / \mathrm{m}, E I=7.6 \mathrm{MN} / \mathrm{m}^{2}, m_{t}=$ $208 \mathrm{~kg} / \mathrm{m}, k 1=4.70 \mathrm{MN} / \mathrm{m}^{2}, k_{2}=48.50 \mathrm{MN} / \mathrm{m}^{2}, k_{1 \mathrm{o}}=100 \mathrm{MN} / \mathrm{m}^{2}, k_{21}=4.9318 \mathrm{MN} / \mathrm{m}^{2}, k_{22}$ $=94.175 \mathrm{MN} / \mathrm{m}^{2}, w o=2 \mathrm{~mm}$ and $Q o=29.981 \mathrm{kN}$ [5]. Regarding the damping, the following damping ratio are considered: $\mathrm{D}_{1 \mathrm{o}}=c_{10} / 2\left(k_{10} m\right)^{1 / 2}$ for the rail pad and $\mathrm{D}_{21}=$ $c_{21} / 2\left(k_{21} m\right)^{1 / 2}, \square_{22}=c_{22} / 2\left(k_{22} m\right)^{1 / 2}$ for the ballast; $\mathrm{D}_{1 \mathrm{o}}=0.2$ and $\mathrm{D}_{21}=\mathrm{D}_{22}=0.25$.

To study the influence of the bilinear characteristic, the linear equivalent model should be used as reference. The equivalent stiffness of the track is obtained with the formula [9]

$$
k_{e}=\frac{1}{4} \frac{Q}{w(0)} \sqrt[3]{\frac{Q}{w(0) E I}}
$$

And the ballast's equivalent stiffness with the formula

$$
\frac{1}{k_{2}}=\frac{1}{k_{e}}-\frac{1}{k_{1 o}}
$$

Where $w(0)$ is the rail deflection under the static load $Q$. 
The deflection of the rail under the static load calculated using the bilinear characteristic model and with the equivalent linear model are presented in the Figure 4.

The equivalent linear model is underestimating the rail deflection close to the application point, in particular when the static load is higher.
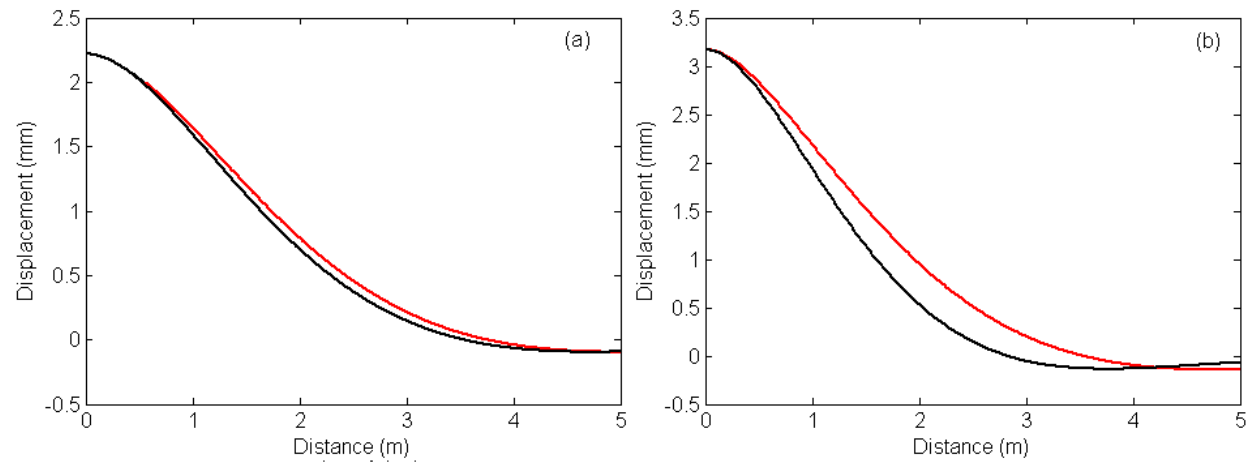

Fig. 4. Rail deflection: (a) static load of $40 \mathrm{kN}$; (b) static load of $112.5 \mathrm{kN}$ : red - bilinear characteristic; black - linear equivalent characteristic.
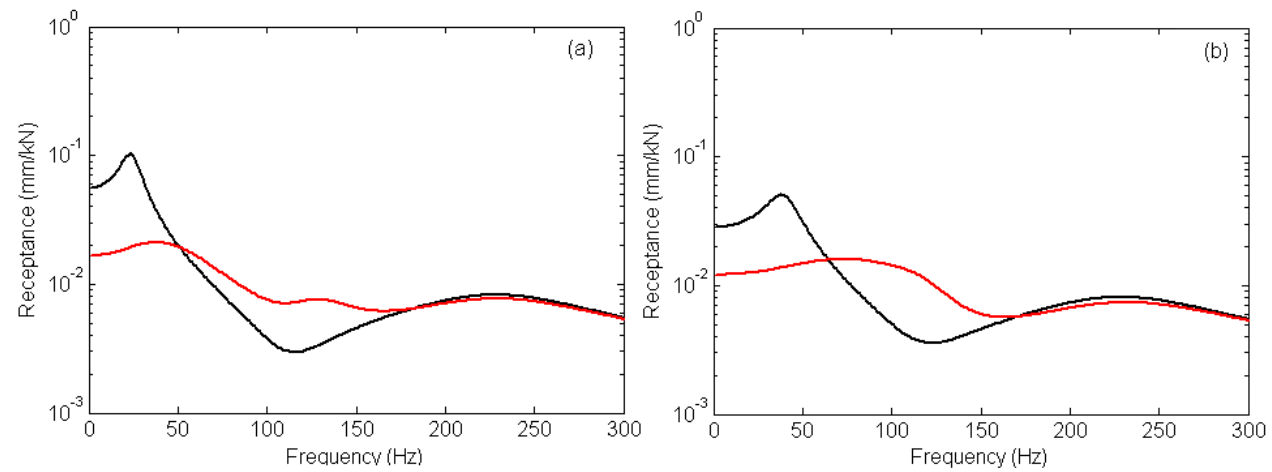

Fig. 5. Rail receptance: (a) static load of $40 \mathrm{kN}$; (b) static load of $112.5 \mathrm{kN}$; red - bilinear characteristic; black - linear equivalent characteristic.
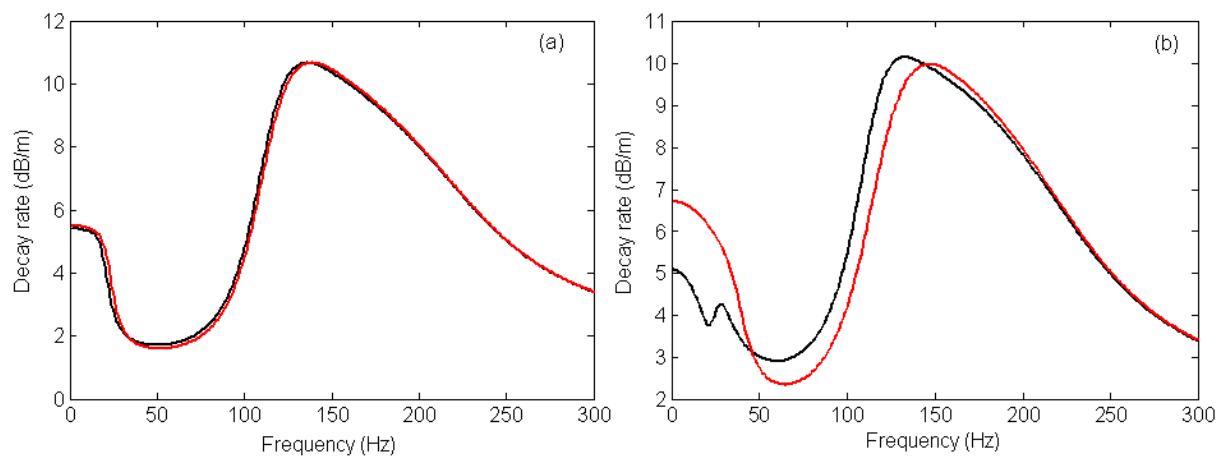

Fig. 6. Decay rate: (a) static load of $40 \mathrm{kN}$; (b) static load of $112.5 \mathrm{kN}$; red - bilinear characteristic; black - linear equivalent characteristic.

The receptance of the track around the application point of the harmonic force, $r(0,0$, $\omega$ ), for the frequencies between 0 and $300 \mathrm{~Hz}$ is presented in the Figure 5 . 
The calculation of the track's receptance has been done for the bilinear characteristic model and for the equivalent linear model for static loads of $40 \mathrm{kN}$ (Figure 5. a) and 112.5 $\mathrm{kN}$ (static load of $225 \mathrm{kN} /$ wheelset) (Figure 5. b). There are notable differences between the two models to frequencies up to $160 \mathrm{~Hz}$.

The decay rate of the vertical vibrations along the rail, calculated in the same conditions as above, is presented in Figure 6. Significant differences between the results obtained with the two models appear with a static load of $112.5 \mathrm{kN}$.

\section{Conclusions}

In this paper, the influence of the bilinear characteristic of the ballast on the dynamic response of the track and on the decay rate of the vibrations, was investigated. The track model is including an infinite beam on a two elastic layers foundation, the first with a linear characteristic and the second one with a bilinear characteristic and an inertial intermediate layer.

The bilinear characteristic of the ballast influences the dynamic response of the track at low and mid frequencies up to approx. $150 \mathrm{~Hz}$.

The ballast's bilinear characteristic influences the decay rate of the track only if the static load on the wheel is high enough.

\section{References}

1. M. Dumitriu, UPB Scie. Bull., Series D: Mech. Eng. 74, 4, 75-90 (2012)

2. M. Dumitriu, I. Sebeşan, Journal of Engineering Science and Technology Review 10, 1, 160-167, (2017)

3. M. A. Spiroiu, IOP Conf. Series: Mat. Scie. and Eng., 147, 1, Article number 012117 , (2016).

4. M. Dumitriu, C. I. Cruceanu, J. of Eng. Sci. and Tech. Review, 10, 6 87-95 (2017)

5. A. Kerr, A. Eberhardt, Rail Int. Mars, 41-54 (1992)

6. T. X. Wu, D. J. Thompson, J Sound Vibr 219, 881-904, (1999)

7. T. Mazilu, Procedia Eng. 199, 2561-2566, (2017)

8. T. Mazilu, Proc. Rom. Acad., Ser. A 10, 139-150 (2009)

9. T. Mazilu, Wheel-Rail Vib. (in Romanian), (MatrixRom, Bucharest, 2008) 